\title{
Mitteilungen
}

\section{Swiss Lung Foundation}

\section{Ewald-Weibel-Förderpreis 2018 für Lungenforschung in der Schweiz}

Die Swiss Lung Foundation und das Institut für Anatomie der Universität Bern verleihen einen Preis von 10000 CHF für ausgezeichnete Leistungen auf dem Forschungsgebiet «Lunge und Atmung» in der Schweiz

Der Preis wird seit 2017 für ein aussichtsreiches, eigenständiges Werk insbesondere von Nachwuchsforschenden verliehen. Die Wahl des Preisträgers / der Preisträgerin erfolgt durch die Preiskommission.

\section{Anforderungen:}

Die Forschung soll in der Regel aus einer schweizerischen Universität, Klinik oder Forschungsanstalt stammen resp. mehrheitlich in der Schweiz entstanden sein.

Die Nominationen sind in deutscher, französischer oder englischer Sprache zu begründen, wobei Englisch bevorzugt wird. Sie sollen das Curriculum vitae mit Publikationsverzeichnis und eine Zusammenfassung des wissenschaftlichen Werdegangs und Werks, mit Bezeichnung der 3-5 wichtigsten Publikationen (nicht mehr als zwei Seiten und in Englisch) enthalten.

Die Unterlagen müssen bis zum 15.12.2017 unter folgender Adresse eingereicht werden: Dr. med. Otto Brändli, Präsident Swiss Lung Foundation, Hömelstrasse 15, 8636 Wald, braendli[at]swisslung.org

\section{Preiskommission:}

Dr. med. Otto Brändli, Präsident Prof. Dr. med. vet. Max Gassmann Prof. Dr. med. Thomas Geiser Prof. Dr. med. Laurent Nicod Prof. Dr. phil. nat. Johannes Schittny

\section{Franco-Regli-Stiftung}

Preis 2017 im Forschungsgebiet von neurodegenerativen Erkrankungen

Die Stiftung verleiht in der Schweiz tätigen Forschern oder Forschungsteams, gleich welcher Nationalität, einen Preis in Höhe von 10000 CHF für eine hervorragende wissenschaftliche Arbeit auf dem Gebiet der neurodegenerativen Erkrankungen.

Berücksichtigt werden Arbeiten aus der Grundlagenforschung oder der klinischen Forschung, die 2016/2017 publiziert oder für eine wissenschaftliche Publikation im Jahre 2017 angenommen wurden. Schweizer Autoren können auch im Ausland entstandene Arbeiten einreichen.

Die Arbeiten sind bis zum 31. Oktober 2017 zusammen mit einem Lebenslauf per E-Mail einzureichen. Erbeten werden zudem 2 Exemplare der publizierten oder zur Publikation angenommenen Arbeit auf Papier.

Adresse:

Cristina Donati-Regli, Mitglied des Stiftungsrates, Brunnadernstrasse 29, 3006 Bern, E-Mail: info[at]fondationfrancoregli.ch, www.fondationfrancoregli.ch 